\title{
Detection of Intrusive Activity in Databases by Combining Multiple Evidences and Belief Update
}

\author{
Suvasini Panigrahi*, Shamik Sural*, and A. K. Majumdar ${ }^{\dagger}$, \\ ${ }^{*}$ School of Information Technology \\ $\dagger$ Department of Computer Science \& Engineering \\ Indian Institute of Technology, Kharagpur, India \\ \{Suvasini.Panigrahi@sit, shamik@sit, akmj@cse\}.iitkgp.ernet.in
}

\begin{abstract}
In this paper, we propose an innovative approach for database intrusion detection which combines evidences from current as well as past behavior of users. It consists of four components, namely, rule-based component, belief combination component, security sensitive history database component and Bayesian learning component. The rule-based component consists of a set of well-defined rules which give independent evidences about a transaction's behavior. An extension of DempsterShafer's theory is used to combine multiple such evidences and an initial belief is computed. First level inferences are made about the transaction depending on this initial belief. Once the transaction is found to be suspicious, belief is updated according to its similarity with malicious or genuine transaction history using Bayesian learning. Experimental evaluation shows that the proposed intrusion detection system can effectively detect intrusive attacks in databases without raising too many false alarms.
\end{abstract}

Index Terms - Database security, Dempster-Shafer theory, Bayesian learning, Intrusion detection, Suspicion score

\section{INTRODUCTION}

Database systems form an indispensable component of the information system infrastructure in most organizations and are adopted as the key data management technology for daily operations and decision making in businesses. Data constituting such databases may contain invaluable sensitive information and organizations manage access to these data meticulously, with respect to both internal as well as external users. Any breach of security or intrusion in these databases perturbs not only a single user but can have devastating consequences on the entire organization [1].

According to a computer crime and security survey conducted by the Computer Security Institute (CSI) [2] in 2005, approximately $45 \%$ of the inquired entities have reported increased unauthorized access to information. The 2007 CSI computer crime and security survey [3] proclaimed financial application fraud as the leading cause of financial loss and found it more than doubled as compared to the loss estimated in the previous year. Another significant cause of loss was system penetration by outsiders. Additional findings in the current year's survey include the fact that, 59\% of all respondents outlined insider abuse as the most prevalent security problem. These figures show the growing sophistication and stealth of attacks on databases. In addition to the substantial financial losses, intrusive attacks can also tarnish reputation of organizations, cause loss of customer confidence and even litigations. Thus, database intrusion detection is a significant problem which needs to be addressed urgently.

Intrusive attacks in databases can be categorized as insider attack and outsider attack depending on the source from which it occurs. An insider attack [4] is one in which unauthorized database transactions are carried out by authorized users within the organization. These attacks are particularly difficult to identify as the perpetrators may have access rights to data and system resources and could be familiar with the database schema and security setup of the organization. Carter and Katz [5] have revealed that the primary security threat comes from internal abuse rather than from outside attacks. In case of outsider attack, malicious transactions are executed by unauthorized users from outside the organization, who may have gained access to the database by exploiting system vulnerabilities.

Traditional database security mechanisms do not assure protection against database vulnerability exploits and are limited in defending security attacks from inside the organization or from unsecured applications. Therefore, protection of data stored in databases from unauthorized accesses or malicious actions is of utmost importance. One mechanism to safeguard the valuable information in these databases is to complement the database system by a powerful intrusion detection system (IDS), which provides an additional layer of security that cannot be assured by classical security mechanisms. These systems aim to detect intrusions as early as possible so that the damage caused by the intrusions is minimized. The possibility of enhancing existing security mechanisms by introducing an effective database IDS constitutes the objective of our work.

The rest of the paper is organized as follows. Section II describes related work on intrusion detection. Section III presents the components of the proposed intrusion detection system along with a description of the methodology. In Section IV, we discuss the results obtained from comprehensive simulation studies. Finally, we conclude in Section V of the paper.

\section{RELATED WORK}

Research on intrusion detection (ID) has been ongoing for more than two decades and several intrusion detection systems have been developed. The previous efforts were mostly focused on network-based and host-based intrusion detection systems [6][7]. Salerno et al. [8] and Kandefer et al. [9] have 
attempted detection of intrusions in the cyber security domain. However, these IDSs are not capable of detecting intrusions in databases as they do not work at the application level. In spite of the significant role of databases in information systems, very limited research has been carried out in the field of database intrusion detection.

Chung et al. [10] present DEMIDS, a misuse detection system for relational database systems. This method assumes that the legitimate users show some level of consistency in using the database system. If this assumption does not hold, it results in a large number of false positives. Lee et al. [11] have described a method to discover intrusions in real-time databases. A security alarm is invoked when a transaction attempts to write a temporal data object that is already updated within a period. Wenhui et al. [12] present a twolayer mechanism to detect intrusions in web-based database systems. The first layer is used to build historical profiles and pre-alarms may be generated due to abnormal behavior. The second layer further verifies the alarms generated by the layer one. Barbara et al. [13] use Hidden Markov Model (HMM) and time series to find malicious corruption of data.

Lee et al. [14] designed a signature-based database intrusion detection system (DIDS) which detects intrusions by matching new SQL statements against a known set of transaction fingerprints. However, generating the complete set of fingerprints for all transactions and maintaining its consistency is a rigorous activity. Moreover, if any of the legitimate transaction fingerprints are missing, it can cause many false alarms. Another relevant mechanism for database intrusion detection was proposed by $\mathrm{Hu}$ et al. [15]. In this approach, the data dependency relationships among the transactions are mined and this information is used to detect anomalies. Transactions not agreeable with any of the mined data dependency rules are identified as malicious.

Bertino et al. [16] developed another DBMS-specific IDS which mines database log files to generate user profiles that model normal user actions and is used to identify intrusions. They associate the IDS with role-based access control (RBAC) for making the approach applicable even for large databases. Srivastava et al. [17] propose a data mining based IDS that considers sensitivity of attributes while mining the dependency rules. They find data dependency among the data items. Transactions that do not follow the mined data dependency rules, are flagged as malicious.

Majority of the IDSs as discussed above, show a lot of variation in their accuracy and efficiency. The main problem faced by most of them is that any attempt to improve the rate of correct detection of intrusion, usually causes a rise in the false alarms as well. One of the motivations of our current research is to address this challenge.

It is well known that every database user has a certain database access pattern, which establishes an activity profile for him. Almost all the existing intrusion detection techniques try to capture these behavioral patterns as rules and check for any violation in subsequent transactions. However, these rules are largely static in nature, and fail in a scenario when the user develops new patterns of behavior that are not yet known to the DIDS. Thus, systems that cannot "learn", may soon become outdated resulting in large number of false alarms. Moreover, new intrusion types, of which the detection systems are unaware, mostly go undetected. The ability to learn enables the system to detect both known and unknown intrusions efficiently. Thus, there is a need for developing intrusion detection systems which can integrate multiple evidences from these rules including patterns of genuine users as well as that of intruders.

In this paper, we propose a comprehensive database intrusion detection system that integrates different types of evidences using an extended Dempster-Shafer theory [18]. Besides combining evidences, we also incorporate learning in our system through application of prior knowledge and observed data on suspicious users. Furthermore, we have taken the sensitivity of database table attributes into consideration since in every database, there are a few attributes which are more important to be tracked for malicious modifications. To the best of our knowledge, this is the first ever attempt to develop a database intrusion detection system using information fusion and Bayesian learning.

\section{PROPOSED APPROACH}

The proposed system is designed to model normal database access behavior and identify malicious transactions submitted to the DBMS by an intruder. It may be abstractly represented as a 5-tuple $\left\langle U, P, \psi, \theta_{L T}, \theta_{U T}\right\rangle$ where:

1) $U=\left\{U_{1}, U_{2}, \ldots, U_{n}\right\}$ is the set of users on which intrusion detection is performed

2) $P=\left\{P\left(U_{1}\right), P\left(U_{2}\right), \ldots, P\left(U_{n}\right)\right\}$ is the set of profiles, where each $P\left(U_{k}\right)$ corresponds to the profile of the user $U_{k}$. Each user profile $P\left(U_{k}\right)$ can be represented as a 12-tuple: 〈user_ID, attrib_ID_seq, loc_ID,time_slot, table_ID_seq, HSWC, HSRC, MSWC, MSRC, LSWC, $L S R C, \rho\rangle$

- user_ID: a number that identifies each user uniquely

- attrib_ID_seq: attribute access sequence in a transaction

- loc_ID: identifies the location where a transaction was carried out

- time_slot: time slot in which a transaction occurs. We have partitioned a day into 48 time slots, each of thirty minutes duration

- table_ID_seq: table access sequence in a transaction

- HSWC (High Sensitive Write Count): number of high sensitive attributes modified in a transaction. Definitions of HSRC (High Sensitive Read Count), MSWC (Medium Sensitive Write Count) MSRC (Medium Sensitive Read Count), LSWC (Low Sensitive Write Count) and LSRC (Low Sensitive Read Count) follow from the above.

3) $\psi\left(T_{j, \rho}^{U_{k}}\right)$ : suspicion score of the $j^{t h}$ transaction $T_{j, \rho}^{U_{k}}$ by user $U_{k}$ with time gap $\rho$ 
4) $\theta_{L T}$ : lower threshold $\left\{0 \leq \theta_{L T} \leq 1\right\}$

5) $\theta_{U T}$ : upper threshold, where $\left\{\left(0 \leq \theta_{U T} \leq 1\right) \wedge\left(\theta_{L T} \leq\right.\right.$ $\left.\left.\theta_{U T}\right)\right\}$

\section{A. System Components}

In the proposed DIDS, a number of rules are used to analyze the deviation of each incoming transaction from the normal profile of the user by assigning basic probabilities to it. The basic probability values are combined to obtain an initial belief by applying extended Dempster-Shafer theory [18]. The initial belief is updated according to its similarity with transaction history using Bayesian learning. In order to meet this functionality, the proposed DIDS is designed with four major components, namely, Rule-based Component (RBC), Belief Combination Component (BCC), Security Sensitive History Database Component (SSHDC) and Bayesian Learning Component (BLC).

1) Rule-based Component: The RBC consists of a number of rules which classify an incoming transaction as malicious with a certain probability. It measures the extent to which a transaction's behavior deviates from the user's normal profile for each new transaction submitted by the user. We briefly discuss two of the rule-based techniques here.

\section{- Sequence Alignment for Deviation Detection $\left(R_{1}\right)$}

A database user normally follows a specific sequence of operations in order to accomplish a particular task. Therefore, sequence can be an effective way for representing user profiles and thus sequence alignment can be used to detect any anomalous activity. Sequence alignment is a technique used to quantify and evaluate similarity between two or more sequences. We use sequence alignment as a tool for comparing database access patterns of genuine users and intruders. As intruders are not entirely familiar with the normal database access patterns of legitimate users, they usually show some inter-transactional as well as intra-transactional deviation in their database access. Thus, most of the intrusive activities can be recognized through an analysis of past database access patterns. Basic Local Alignment Search Tool (BLAST) [19] is one of the most popular heuristic approaches for sequence alignment, which is used in our DIDS for comparing sequence information.

Each new transaction is passed through the RBC and the new attribute sequence is aligned with each of the normal profile sequences. The degree of dissimilarity $\left(d_{s}\right)$ is determined based on the dissimilarity between the new sequence and the user's normal profile sequences. We use a simple scoring system to evaluate the degree of dissimilarity. A unit match score $\delta(0<\delta \leq 1)$ is assigned to each matched element and a unit mismatch score $\delta^{\prime}\left(0<\delta^{\prime} \leq 1\right)$ to each mismatched element. Let $L$ be the length of the new sequence and $M$ be the number of matches with the aligned good sequence. The degree of dissimilarity $\left(d_{s}\right)$ is then evaluated by the following expression:

$$
d_{s}= \begin{cases}\frac{\delta^{\prime}(L-M)-\delta M}{L} & \begin{array}{l}
\text { if } \delta^{\prime}(L-M)>\delta M \\
\text { otherwise }
\end{array}\end{cases}
$$

In the current work, we use attrib_ID_seq as the transactional feature for sequence alignment deviation detection. The algorithm can be extended to include other transactional features as well.

- Spatio-Temporal Outlier Detection $\left(R_{2}\right)$

Similar transactions carried out by a user at certain certain location and time can be visualized as part of a cluster. Such observation is known as a spatio-temporal point which is scale-dependent on space and time. It may be noted that such spatio-temporal coordinates are especially meaningful in the context of mobile computing. We mine out the normal spatio-temporal activity patterns of each user and use it for detection of malicious activities in databases. Since an intruder is not having complete knowledge regarding the spatio-temporal access patterns of users, some deviation is normally seen from the user's profile, his transactions can be detected as exceptions to the cluster - a process known as spatio-temporal outlier detection. A spatio-temporal outlier ( $S T$-outlier) can be defined as a spatio-temporal referenced object whose thematic attribute values are significantly different from those of other spatially and temporally referenced objects in its spatial and temporal neighborhood.

We utilize an approach based on the distance-based outlier ( $D B$-outlier) detection technique proposed in [20], to filter out spatio-temporal outliers ( $S T$-outliers). Let $N$ be the number of objects in the input dataset $T$ and let $D F$ be the underlying distance function that gives the distance between any pair of objects in $T$. An object $O$ in a dataset $T$ is considered to be a $D B(p, d)$ outlier if at least a fraction $p$ of the objects in $T$ lie at a distance greater than $d$ from $O\left(d\right.$-neighborhood denoted by $\left.d_{N}\right)$. Let $M$ represent the maximum number of data points within an outlier's $d_{N}$ (i.e., $M=N(1-p)$ ). The clusters can be formed by using different attributes, although in the current work, we use the attributes $\left\langle l o c \_I D, t i m e \_s l o t, t a b l e \_I D \_s e q\right\rangle$ for generating $S T$-outliers. We compute the distance function $D F$ by applying Euclidean distance, which can be expressed as follows:

$$
D F=\sqrt{(\text { loc_diff })^{2}+(\text { time_diff })^{2}+(\text { tdist_diff })^{2}}
$$

where loc_diff: distance between current transaction location and the user's normal profile transaction location, time_diff: distance between current transaction time slot and the user's normal profile time slot, tdist_diff: schema distance between current transaction table_ID_seq and the user's normal profile table_ID_seq.

For computing tdist_diff, we use a distance measure similar to that suggested in [10]. We assume a database schema $S$ with a set $R S$ of relation schemas. Attributes 
are structurally close if they belong to the same relation or can be related by exploiting a sequence of foreign key dependencies. Suppose two attributes $a_{i} \in r_{1}, a_{j} \in$ $r_{2}$ where $r_{1}, r_{2} \in R S$. The pairwise schema distance between $a_{i}$ and $a_{j}$, denoted by $P S_{\_} d i s t$ is defined as:

$$
P S \_D i s t\left(a_{i}, a_{j}\right)=\frac{S D\left(r_{1}, r_{2}\right)}{\max \left\{S D\left(r_{k}, r_{l}\right) \mid r_{k}, r_{l} \in R S\right\}}
$$

where $S D\left(r_{1}, r_{2}\right)$ is the shortest distance between $r_{1}$ and $r_{2}$. Given a set of attributes $A=\left\{a_{1}, a_{2}, \ldots, a_{n}\right\} \subseteq$ attributes $(S)$, the schema distance function denoted by tdist_diff, is defined as:

$$
\operatorname{tdist\_ diff}\left(a_{1}, \ldots, a_{n}\right)=\operatorname{avg}\left\{P S \_d i s t\left(a_{i}, a_{j}\right)\right\}
$$

We measure the extent of deviation of an incoming transaction by its degree of $S T_{-}$outlierness. Suppose $D F_{\text {avg }}\left(T_{j, \rho}^{U_{k}}\right)$ and $D F_{\max }\left(T_{j, \rho}^{U_{k}}\right)$ denote average distance and maximum distance of an outlier transaction $T_{j, \rho}^{U_{k}}$ from the set of existing clusters in $C^{\prime}$ respectively. The degree of $S T_{-}$outlierness $\left(d_{S T O}\right)$ of $T_{j, \rho}^{U_{k}}$ is then given by:

$$
d_{S T O}= \begin{cases}\frac{D F_{a v g}\left(T_{j, \rho}^{U_{k}}\right)}{D F_{\max }\left(T_{j, \rho}^{U_{k}}\right)} & \text { if }\left|d_{N}\right| \leq M \\ 0 & \text { otherwise }\end{cases}
$$

Each of these rules $R_{1}$ and $R_{2}$ gives independent evidences about a transaction's behavior, which are combined by the belief combination component of our DIDS. Though we have used two specific techniques as rules in the current work, functionality of the RBC component can be further enriched by incorporating new rules as deemed appropriate in an organization.

2) Belief Combination Component: The role of the BCC is to combine evidences from the rules $R_{1}$ and $R_{2}$ and compute an initial belief for each transaction submitted to the DIDS. It may be noted that some attempts have been made to apply Dempster-Shafer theory (DST) [21] to computer security [22][23]. We have earlier proposed a novel way of detecting credit card fraud using DST [24]. The basic DST is a mathematical theory of evidence based on belief functions and plausible reasoning. It assumes a Universe of Discourse $(U D)$, also called the Frame of Discernment, which is a set of mutually exclusive and exhaustive possibilities. Two basic probability assignments (BPAs) $m_{1}(h)$ and $m_{2}(h)$ are combined by the Dempster's rule [25] as follows:

$$
m(h)=m_{1}(h) \oplus m_{2}(h)=X \sum_{x \cap y=h} m_{1}(x) m_{2}(y)
$$

where, $X$ is the normalization constant defined by the following Eq. (7):

$$
\begin{gathered}
X=\frac{1}{K} \\
K=1-\sum_{x \cap y=\phi} m_{1}(x) m_{2}(y)
\end{gathered}
$$

However, the basic DST has some major drawbacks. The normalization constant in the Dempster's combination rule (Eq. (6)) has the effect of completely ignoring conflict and consequently, this operation will yield counterintuitive results in the face of significant conflict in certain contexts. To solve this problem, we have employed the Extended DempsterShafer theory (EDST) proposed by Campos et al. [18] that presents a new improved rule for combining evidences. EDST overcomes the above mentioned pitfalls by assigning the beliefs according to the degree of conflict between the evidences and the remaining belief to the environment rather than the common hypothesis. The conflict between two belief functions $b e l_{1}$ and $b e l_{2}$, denoted by $C o n\left(b e l_{1}, b e l_{2}\right)$ is given by the logarithm of the normalization constant as follows:

$$
\text { Con }\left(\text { bel }_{1}, \text { bel }_{2}\right)=\log (X)
$$

The modified Dempster's combination rule automatically incorporates the uncertainty coming form the conflicting evidences which is given by the following Eq. (10):

$$
m(h)=m_{1}(h) \oplus m_{2}(h)=\frac{X \sum_{x \cap y=h} m_{1}(x) m_{2}(y)}{1+\log \left(\frac{1}{K}\right)}
$$

EDST provides a rule for computing the confidence measures of three states of knowledge: intrusion $(I)$, ᄀintrusion $(\neg I)$ and suspicious (unknown) based on the data from new as well as old evidence. Hence, we use EDST for combining evidences for this problem. The $U D$ consists of two possible values for any suspected transaction $T_{j, \rho}^{U_{k}}$ which is given as $U D=\{I, \neg I\}$. For this $U D$, the power set has three possible elements - hypothesis $h=\{I\}$ implying that $T_{j, \rho}^{U_{k}}$ is intrusive, hypothesis $\bar{h}=\{\neg I\}$ that it isn't, and universe hypothesis $U D$ that $T_{j, \rho}^{U_{k}}$ is suspicious. The basic probability assignments (BPAs) for the two rules $R_{1}$ and $R_{2}$ can now be given as follows:

- BPA for $R_{1}$ : For a transaction in which attrib_ID_seq does not match completely with the normal profile attrib_ID_seq, we make the following basic probability assignments using the degree of dissimilarity $\left(d_{s}\right)$ given by Eq. (1):

$$
\begin{aligned}
& m_{1}(h)=\frac{\delta^{\prime}(L-M)-\delta M}{L} \\
& m_{1}(\bar{h})=0 \\
& m_{1}(U D)=1-\left(\frac{\delta^{\prime}(L-M)-\delta M}{L}\right)
\end{aligned}
$$

- BPA for $R_{2}$ : For a transaction detected as an $S T$-outlier, we make the following basic probability assignments using the degree of $S T_{-}$outlierness $\left(d_{S T O}\right)$ given by Eq. (5):

$$
\begin{aligned}
& m_{2}(h)=\frac{D F_{a v g}\left(T_{j, \rho}^{U_{k}}\right)}{D F_{\max }\left(T_{j, \rho}^{U_{k}}\right)} \\
& m_{2}(\bar{h})=0 \\
& m_{2}(U D)=1-\left(\frac{D F_{a v g}\left(T_{j, \rho}^{U_{k}}\right)}{D F_{\max }\left(T_{j, \rho}^{U_{k}}\right)}\right)
\end{aligned}
$$


The zero in the BPA of $\bar{h}$ in Eqs. (11) and (12) means that neither of the rules $R_{1}$ and $R_{2}$ gives any support to the belief that transaction $T_{j, \rho}^{U_{k}}$ is genuine. Following Eq. (10), the combined belief of $R_{1}$ and $R_{2}$ in $h$ is expressed as:

$$
P(h)=m_{1}(h) \oplus m_{2}(h)
$$

Based on the initial belief $P(h)$, a transaction can be initially classified as legitimate, malicious or suspicious.

3) Security Sensitive History Database Component: Large amounts of history database transactions are collected and warehoused in the SSHDC for extracting useful information about database usage which facilitates the detection of any malicious activity in the database. Once a transaction from a user is labeled as suspicious, further transactions from this particular user are permitted but his each new transaction is investigated by the DIDS. This is done to avoid troubling the legitimate users making occasional high level of activity. For accomplishing this, we have built a legitimate transactions history (LTH) for individual users from their past behavior and a generic malicious transactions history (MTH) from different types of past intrusive data. It should be noted that we build MTH from history data about past intrusive behaviors detected by any organization. However, for a new user no history is available. In such situations, the proposed model reduces to an anomaly based detection system that combines evidences from the rule-based component.

In recent years, database size has grown considerably in terms of the number of tuples (objects) and number of attributes (fields) in the database [26]. However, in every database, there are a few attributes that are more important to be tracked for malicious modifications or leakage as compared to other attributes. By grouping the attributes according to the relative order of importance based on their sensitivity, it becomes comparatively easier to track only those sensitive attributes whose modification or leakage has larger impact on the database security.

The attributes are categorized into the following three sensitivity levels - High Sensitivity $(H S)$, Medium Sensitivity $(M S)$ and Low Sensitivity $(L S)$. Also, modification (write) of an attribute of a particular sensitivity level is considered more important than accessing (read) the same attribute, from database integrity point of view. For a given schema, we define six types of attribute operations $(o p)$ : High Sensitive Write $(H S W)$, High Sensitive Read $(H S R)$, Medium Sensitive Write $(M S W)$, Medium Sensitive Read $(M S R)$, Low Sensitive Write $(L S W)$ and Low Sensitive Read $(L S R)$, based on their relative order of importance. The weight of a transaction is same as the weight of the most sensitive operation applied on its attribute access sequence. Any behavioral deviation of the current activity is monitored by keeping track of the time_gap $(\rho)$ from the previous transaction by the same user along with the frequency of the most sensitive operation in that time_gap.

To capture the frequency of the most sensitive operation in a specific time_gap for a transaction, we divide the time $e_{-}$ap $(\rho)$ into four units such that, $\rho \in\{1,2,3,4\}$ where $\rho=1 \Rightarrow 0<$ time $_{-}$gap $\leq 8$,

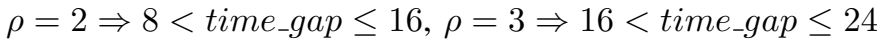
and $\rho=4 \Rightarrow$ time_gap $_{-}>24$. We define 24 mutually exclusive and exhaustive events $D_{\text {op } \rho}$ by considering the four time_gap units $(\rho)$ and the six types of operations $(o p)$ as discussed above. The set of events is expressed as: $D_{o p \rho}=$ $\left\{D_{H S W 1}, D_{H S W 2}, D_{H S W 3}, D_{H S W 4}, \ldots, D_{L S R 3}, D_{L S R 4}\right\}$. The event $D_{H S W 1}$ is defined as the occurrence of a transaction $T_{j, \rho}^{U_{k}}$ by the same user $U_{k}$ in which an $H S W$ operation is performed at $\rho=1$ which can be represented as:

$$
D_{H S W 1}=\operatorname{True} \mid\left\{\exists T_{j, \rho}^{U_{k}} \wedge(\text { op }=H S W \wedge(\rho=1))\right\}
$$

Similarly, the events $D_{H S W 2}, D_{H S W 3}$ and $D_{H S W 4}$ can be expressed as:

$$
\begin{aligned}
& D_{H S W 2}=\operatorname{True} \mid\left\{\exists T_{j, \rho}^{U_{k}} \wedge(\text { op }=H S W \wedge(\rho=2))\right\} \\
& D_{H S W 3}=\operatorname{True} \mid\left\{\exists T_{j, \rho}^{U_{k}} \wedge(\text { op }=H S W \wedge(\rho=3))\right\} \\
& D_{H S W 4}=\operatorname{True} \mid\left\{\exists T_{j, \rho}^{U_{k}} \wedge(\text { op }=H S W \wedge(\rho=4))\right\}
\end{aligned}
$$

The definition of the remaining events follows from the above. It may be noted that, we chose the above definitions of $D_{o p \rho}$ to handle frequent as well as infrequent users during experimentation. Other values could be similarly defined.

We next compute $P\left(D_{o p \rho} \mid h\right)$ and $P\left(D_{o p \rho} \mid \bar{h}\right)$ from the MTH and the LTH respectively. $P\left(D_{o p \rho} \mid h\right)$ measures the probability of occurrence of $D_{o p \rho}$ given that a transaction is originating from an intruder and $P\left(D_{o p \rho} \mid \bar{h}\right)$ measures the probability of occurrence of $D_{o p \rho}$ given that it is genuine, which are given by the following expressions:

$$
\begin{gathered}
P\left(D_{\text {op } \rho} \mid h\right)=\frac{\#\left(\text { Occurrences of } D_{\text {op } \rho} \text { in } M T H\right)}{\sum_{\rho=1}^{4} \#\left(\text { Occurrences of } D_{\text {op } \rho} \text { in } M T H\right)} \\
P\left(D_{\text {op } \rho} \mid \bar{h}\right)=\frac{\#\left(\text { Occurrences of } D_{\text {op } \rho} \text { by } U_{k} \text { in } L T H\right)}{\sum_{\rho=1}^{4} \#\left(\text { Occurrences of } D_{\text {op } \rho} \text { by } U_{k} \text { in } L T H\right)}
\end{gathered}
$$

We have created two look-up tables MFT (Malicious Frequency Table) and LFT (Legitimate Frequency Table) to maintain the values of $P\left(D_{o p \rho} \mid h\right)$ and $P\left(D_{o p \rho} \mid \bar{h}\right)$ respectively. Using Eqs. (18) and (19), $P\left(D_{o p \rho}\right)$ can be computed as follows:

$$
P\left(D_{\text {op } \rho}\right)=P\left(D_{\text {op } \rho} \mid h\right) P(h)+P\left(D_{\text {op } \rho} \mid \bar{h}\right) P(\bar{h})
$$

The initial belief $P(h)$ of Eq. (13) can be updated by using Bayes rule after getting the new information $D_{o p \rho}$ from the SSHDC. We update the SSHDC frequently in order to retain the accuracy of the DIDS, thus reducing the number of false alarms. SSHDC update is an offline procedure.

4) Bayesian Learning Component: Bayesian learning is a tool to measure evidences supporting alternative hypotheses and arrive at optimal decisions. We use Bayesian learning to update the suspicion score $(\psi)$ of a transaction in the light of the new evidence $D_{o p \rho}$ from the SSHDC. $\psi$ is the probability that the current transaction is malicious. Bayes rule gives 
the mathematical formula for belief revision, which can be expressed as follows:

$$
P\left(h \mid D_{\text {op } \rho}\right)=\frac{P\left(D_{\text {op }} \mid h\right) P(h)}{P\left(D_{\text {op }} \mid h\right) P(h)+P\left(D_{\text {op }} \mid \bar{h}\right) P(\bar{h})}
$$

The goal of Bayesian learning is to find the most probable hypothesis $h_{\text {map }}$ given the training data. This is known as the Maximum A Posteriori Hypothesis (MAP Hypothesis) which can be expressed as:

$$
h_{\text {map }}=\max _{h \in H} P\left(h \mid D_{\text {op }}\right)
$$

Thus, for each hypothesis $h$ in the hypothesis space $H$, we calculate the posterior probability $P\left(h \mid D_{\text {op } \rho}\right)$ and $P\left(\bar{h} \mid D_{\text {op } \rho}\right)$ by using Bayes rule and then output the hypothesis with the highest posterior probability as $h_{\text {map }}$. The database intrusion detection problem has the following two hypotheses, $h: I$ and $\bar{h}: \neg I$. By substituting the values obtained from Eqs. (13), (18) and (19) in Eq. (21), the posterior probability for hypothesis $h: I$ is given as:

$$
P\left(I \mid D_{\text {op } \rho}\right)=\frac{P\left(D_{\text {op }} \mid I\right) P(I)}{P\left(D_{\text {op }} \mid I\right) P(I)+P\left(D_{\text {op }} \mid \neg I\right) P(\neg I)}
$$

Similarly, the posterior probability for hypothesis $\bar{h}: \neg I$ is given as:

$$
P\left(\neg I \mid D_{\text {op } \rho}\right)=\frac{P\left(D_{\text {op }} \mid \neg I\right) P(\neg I)}{P\left(D_{\text {op } \rho} \mid I\right) P(I)+P\left(D_{\text {op } \rho} \mid \neg I\right) P(\neg I)}
$$

where $I$ signifies intrusion. Depending on which of the two posterior values is greater, future actions are decided by the DIDS.

\section{B. Methodology}

The mechanism for the detection of malicious database transactions has been depicted in the block diagram of Fig. 1. Each incoming transaction is first examined by the rulebased component of the system. In our model, decision making occurs in two stages. In the first stage, the basic probability values $B P A\left(R_{1}\right)$ and $B P A\left(R_{2}\right)$ assigned by the $\mathrm{RBC}$ are combined using the BCC to get the initial belief $P(h)$ for the transaction. If $P(h)<\theta_{L T}$, the transaction is considered to be genuine and is allowed to go through. On the other hand, if $P(h)>\theta_{U T}$ then the transaction is declared as malicious and manual confirmation can be made with the legitimate user. In case $\theta_{L T} \leq P(h) \leq \theta_{U T}$, the transaction is allowed but the user ID corresponding to the user is labeled as suspicious. If this is the first suspicious transaction carried out by the user, then the corresponding user $I D$ is inserted into a suspect_table. The DIDS then waits until the next transaction occurs using the same user ID.

When the next transaction occurs for the same user, it is again passed through the DIDS. In case the transaction is found to be suspicious, it is inserted into the suspect_table. Since each transaction is time stamped, from the time_gap $(\rho)$ between the current and previous transactions and the most sensitive operation applied on the attrib_ID_seq in the current transaction, our detection system determines which event $E$ has occurred out of the twenty four $D_{o p \rho}$ s and retrieves the corresponding $P(E \mid h)$ and $P(E \mid \bar{h})$ values from the tables MFT and LFT, respectively. The posterior beliefs $P(h \mid E)$ and $P(\bar{h} \mid E)$ are next computed using Eq. (23) and Eq. (24) and MAP hypothesis (Eq. (22)) is applied.

$P(h \mid E)$ and $P(\bar{h} \mid E)$ are the updated beliefs about the last transaction by the user based on the evidence from SSHDC and previous round suspicion score $\psi$ (last round). Since for the second suspicious transaction on a user, there is no $\psi$ (last round), the $P(h)$ value of the first round is itself taken as $\psi$ (last round) and posterior beliefs are computed based on this value. If $P(h \mid E) \geq P(\bar{h} \mid E)$, then the second stage belief combination takes place to obtain the suspicion score $\psi$ (current round) by combining $P(h \mid E)$ and current round $P(h)$. The current round $\psi$ value is inserted into the suspect table at the end of each round unless the suspicious score falls below $\theta_{L T}$ or goes above $\theta_{U T}$.

\section{EXPERIMENTS AND RESULTS}

In this section, the results from an experimental evaluation of the proposed approach is outlined and its performance is illustrated. We have devised an extensive simulator which can handle various real life scenarios that would be normally experienced in actual database applications. Firstly, any realworld database application contains malicious transactions interspersed with regular genuine transactions. Secondly, the genuine transactions are mostly consistent for a given user profile and are scale-dependent on space and time. Thirdly, the genuine transactions and malicious transactions are independent events generated by two different parties and they have separate arrival rates. We capture such real life situations using a Markov Modulated Poisson Process (MMPP). The MMPP consists of a legitimate state $L$ and malicious state $M$ with arrival rates $\lambda_{L}$ and $\lambda_{M}$ respectively. Mixing of legitimate and malicious transactions is controlled by the transition between $L$ and $M$ states. Transition from $L$ to $M$ takes place with probability $q_{L M}$ and from $M$ to $L$ with probability $q_{M L}$.

\section{A. Choice of Design Parameters}

From the discussions in Section III, it is obvious that the effectiveness of the proposed system is dependent on the two parameters $\theta_{L T}$ and $\theta_{U T}$. In order to determine the impact of these parameters on our detection system, eight different simulator settings (SS1 to SS8) were chosen by varying different simulation parameters as shown in Table I. Standard metrics are used to study the performance of the system under different test cases. True positives (TP) are the intrusive transactions caught by the system and false positives (FP) are the genuine transactions labeled as intrusive (also called false alarms).

The results shown in Table II represent average of the results obtained for each of the eight simulator settings of Table I. It is found that at $\theta_{L T}=0.3$ and $\theta_{U T}=0.7$, the difference between TP and FP is the maximum. We make this as our design choice and these threshold values are kept fixed for the rest of the experiments. 


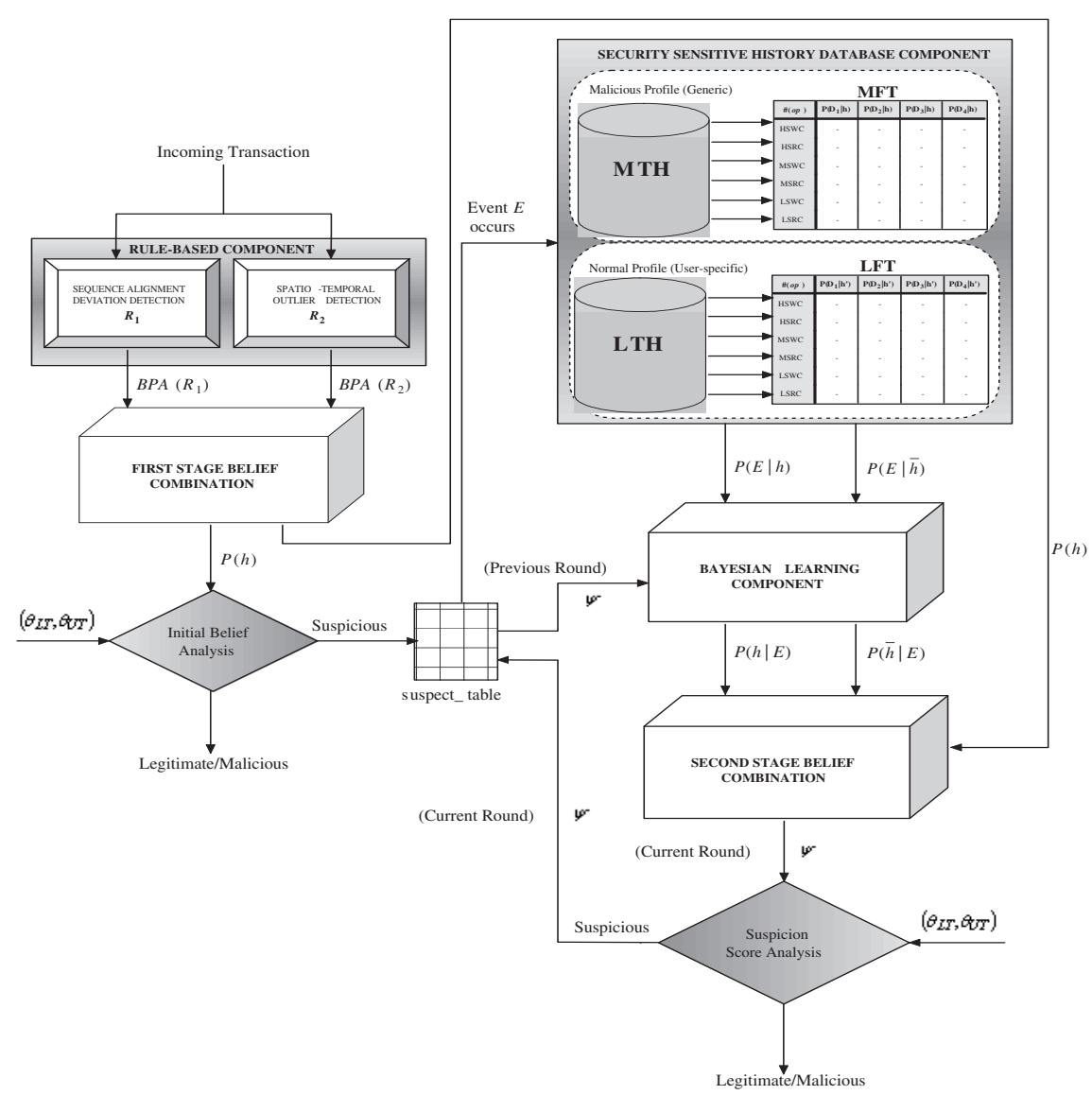

Fig. 1. Block Diagram of the proposed Database Intrusion Detection System

TABLE I

SimUlator SETTINGS FOR ARRIVAL RATE VARIATIONS

\begin{tabular}{|c|c|c|c|c|}
\hline Simulator Setting & $q_{L M}$ & $q_{M L}$ & $\lambda_{L}$ & $\lambda_{M}$ \\
\hline SS1 & 50 & 50 & 1 & 4 \\
\hline SS2 & 15 & 50 & 1 & 4 \\
\hline SS3 & 15 & 70 & 1 & 4 \\
\hline SS4 & 10 & 80 & 1 & 4 \\
\hline SS5 & 10 & 80 & 1 & 2 \\
\hline SS6 & 10 & 90 & 3 & 1 \\
\hline SS7 & 5 & 96 & 4 & 1 \\
\hline SS8 & 5 & 99 & 8 & 1 \\
\hline
\end{tabular}

\section{B. Performance Analysis}

We study the performance of the proposed DIDS against the change in the percentage of overlap between the malicious query set and the legitimate query set. It is evident from the plot shown in Fig. 2 that the TP rate gradually decreases with increase in the percentage of overlapping queries. The performance is worst (lowest TP) at the point of complete overlap. The reason is that, with increase in the percentage of overlap, the similarity among malicious query set and genuine query set increases which makes it difficult to distinguish between them leading to degraded performance. It is seen that FP rate also reduces with rise in the percentage of overlapping queries, but the reduction is slower.

We next compare the performance of our proposed database
TABLE II

VARIaTion of MEAN TP/MEAN FP (\%) With $\theta_{L T}$ AND $\theta_{U T}$

\begin{tabular}{|c|c|c|c|c|}
\hline \multirow{2}{*}{$\theta_{U T}$} & \multicolumn{4}{|c|}{$\theta_{L T}$} \\
\cline { 2 - 5 } & 0.2 & 0.25 & 0.3 & 0.35 \\
\hline 0.7 & $84.5 / 9.2$ & $83.4 / 8.7$ & $\mathbf{8 3 . 2 / 5 . 1}$ & $79 / 5$ \\
\hline 0.75 & $82 / 8.5$ & $81.2 / 7.5$ & $78.6 / 5$ & $77.5 / 4.8$ \\
\hline 0.8 & $81.4 / 7$ & $80 / 5.4$ & $78 / 4.7$ & $74 / 3.8$ \\
\hline 0.85 & $78 / 5$ & $77.1 / 4$ & $76.3 / 3.5$ & $73.6 / 2.3$ \\
\hline
\end{tabular}

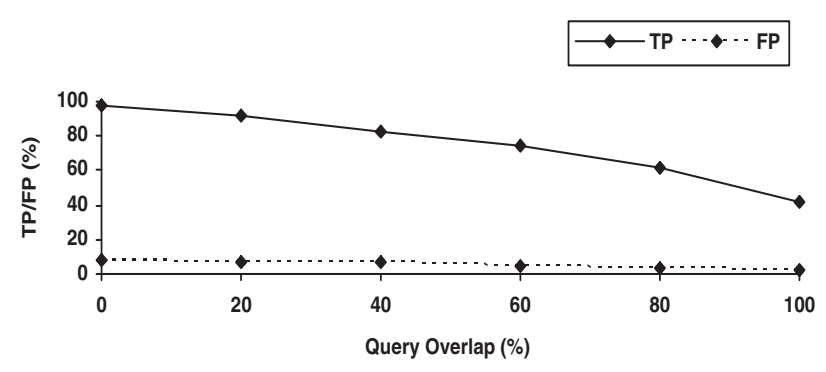

Fig. 2. Variation of TP/FP with the percentage of overlap between the malicious query set and the legitimate query set 
intrusion detection system (PDIDS) with another DIDS proposed by $\mathrm{Hu}$ et al. (DDIDS) [15], which uses data dependency relationships. We compute TP and FP at each simulator setting of Table I for both the systems. Fig. 3 shows that PDIDS has significantly higher detection rate (TP) as compared to DDIDS. However, in terms of FP, performance of both the systems are comparable.

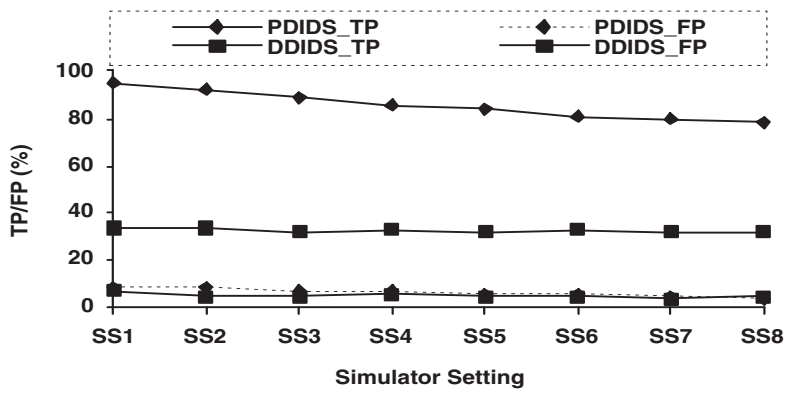

Fig. 3. Variation of TP/FP with different simulator settings for PDIDS and DDIDS

\section{CONCLUSiOnS}

In this paper, we have developed a new approach for detecting intrusions in databases by fusion of information sources and use of belief update. The modified Dempster's rule is applied to combine multiple evidences from the rule-based component for computation of initial belief about each incoming transaction. Belief is updated by means of Bayesian learning using history database of both legitimate users as well as intruders. Moreover, we have also considered three different sensitivity levels of attributes which manifest their relative order of significance for malicious modifications. The experiments yielded up to $98 \%$ TP and less than $10 \%$ FP. While combining rules using extended Dempster-Shafer theory accelerates detection rate compared to the existing techniques, Bayesian learning helps to further improve the system accuracy.

Acknowledgments: This work is partially supported by a research grant from the Department of Information Technology, Ministry of Communication and Information Technology, Government of India, under Grant No. 12(34)/04-IRSD dated 07/12/2004.

\section{REFERENCES}

[1] E. Bertino, R. Sandhu, "Database Security - Concepts, Approaches, and Challenges", IEEE Transactions on Dependable and Secure Computing, Vol. 2, No. 1, Pages 2-19, Jan.-March 2005.

[2] L. A. Gordon, M. P. Loeb, W. Lucyshyn and R. Richardson, "2005 CSI/FBI Computer Crime and Security Survey", http://i.cmpnet.com/gocsi/db_area/pdfs/fbi/FBI2005.pdf.

[3] R. Richardson, "2007 CSI Computer Crime and Security Survey", http://i.cmpnet.com/v2.gocsi.com/pdf/CSISurvey2007.pdf.

[4] S. Furnell, "Enemies Within: The Problem of Insider Attacks", Computer Fraud \& Security, Vol. 2004, Pages 6-11, July 2004.

[5] D. L. Carter and A. J. Katz, "Computer Crime: An Emerging Challenge for Law Enforcement", FBI Law Enforcement Bulletin, http://www.fbi.gov/publications/leb/1996/dec961.txt, Pages 1-8, Dec. 1996.
[6] A. Lazarevic, V. Kumar, J. Srivastava, "Intrusion Detection: A Survey", In Managing Cyber Threats: Issues, Approaches, and Challenges, Springer, Vol. 5, Pages 19-78, 2005

[7] A. J. Hoglund, K. Hatonen and A. S. Sorvari, "A Computer Host-Based User Anomaly Detection System Using the Self-Organizing Map", In Proceedings of the IEEE-INNS-ENNS International Joint Conference on Neural Networks (IJCNN), Vol. 5, 2000.

[8] J. J. Salerno, G. Tadda, M. Hinman and S. Gorton, "Achieving Situation Awareness in a Cyber Environment", In Proceedings of the Workshop on Situation Management, SIMA 2005, In conjunction with IEEE MILCOM 2005, October 2005.

[9] M. Kandefer, S. Shapiro, A. Stotz, and M. Sudit, "Symbolic Reasoning in the Cyber Security Domain", In Proceedings of the MSS 2007 National Symposium on Sensor and Data Fusion, June 2007.

[10] C. Y. Chung, M. Gertz and K. Levitt, "DEMIDS: A Misuse Detection System for Database Systems", In Proceedings of the Integrity and Internal Control in Information System, Pages 159-178, 1999.

[11] V. Lee, J. Stankovic and S. Son, "Intrusion Detection in Real Time Databases via Time Signatures", In Proceedings of the $6^{\text {th }}$ IEEE RealTime Technology and Applications Symposium (RTAS), Pages 124-133, 2000.

[12] S. Wenhui and T. Tan, "A Novel Intrusion Detection System Model for Securing Web-based Database Systems", In Proceedings of the $25^{\text {th }}$ Annual International Computer Software and Applications Conference (COMP-SAC), Pages 249-254, 2001.

[13] D. Barbara, R. Goel, and S. Jajodia, "Mining Malicious Data Corruption with Hidden Markov Models", In Proceedings of the $16^{\text {th }}$ Annual IFIP WG 11.3 Working Conference on Data and Application Security, Pages 175-189, July 2002.

[14] S.Y. Lee, W. L. Low and P. Y. Wong, "Learning Fingerprints for a Database Intrusion Detection System", In Proceedings of the $7^{\text {th }}$ European Symposium on Research in Computer Security, Pages 264-280, 2002.

[15] Y. Hu and B. Panda, "A Data Mining Approach for Database Intrusion Detection", In Proceedings of the ACM Symposium on Applied Computing, Pages 711-716, 2004.

[16] E. Bertino, E. Terzi, A. Kamra and A. Vakali, "Intrusion Detection in RBAC-administered Databases", In Proceedings of the $21^{\text {st }}$ Annual Computer Security Applications Conference (ACSAC), Pages 170-182, Dec. 2005.

[17] A. Srivastava, S. Sural and A.K. Majumdar, "Weighted Intra transactional Rule Mining for Database Intrusion Detection", In Proceedings of the Pacific-Asia Knowledge Discovery and Data Mining (PAKDD), Lecture Notes in Artificial Intelligence, Springer Verlag, Pages 611-620, 2006

[18] F. Campos and S. Cavalcante, "An Extended Approach for DempsterShafer Theory", In Proceedings of the IEEE International Conference on Information Reuse and Integration, Pages 338-344, 2003.

[19] S. F. Altschul, W. Gish, W. Miller, W. Myers and J. Lipman, "Basic Local Alignment Search Tool", Journal of Molecular Biology, Vol. 215, Pages 403-410, 1990.

[20] E. M. Knorr, R. T. Ng and V. Tucakov, "Distance-based Outliers: Algorithms and Applications", International Journal on Very Large Data Bases, Vol. 8, Pages 237-253, Feb. 2000.

[21] G. Shafer, "A Mathematical Theory of Evidence", Princeton Univ. Press, Princeton, 1976.

[22] Y. Wang, H. Yang, X. Wang and R. Zhang, "Distributed Intrusion Detection System Based on Data Fusion Method", In Proceedings of the $5^{\text {th }}$ World Congress on Intelligent Control and Automation, Pages 4331-4334, June 2004

[23] T. M. Chen and V. Venkataramanan, "Dempster-Shafer Theory for Intrusion Detection in Ad Hoc Networks", In Proceedings of the IEEE Internet Computing, Pages 35-41, Nov/Dec 2005.

[24] S. Panigrahi, A. Kundu, S. Sural and A. K. Majumdar, "Credit Card Fraud Detection: A Fusion Approach using Dempster-Shafer Theory and Bayesian Learning", Information Fusion (Special Issue on Information Fusion for Computer Security), 2009 (to appear).

[25] K. Sentz, "Combination of Evidence in Dempster-Shafer Theory", Sandia National Laboratories, US Department of Energy, http://www.sandia.gov/epistemic/Reports/SAND2002-0835.pdf, 8 Dec. 2007.

[26] U. Fayyad, G. P. Shapiro and P. Smyth, "The KDD Process for Extracting Useful Knowledge from Volumes of Data", Communications of the ACM, Vol. 39, Pages 27-34, 1996. 